\title{
Complexity of curves
}

\author{
by
}

Udayan B. Darji (Louisville, KY) and Alberto Marcone (Udine)

\begin{abstract}
We show that each of the classes of hereditarily locally connected, finitely Suslinian, and Suslinian continua is $\Pi_{1}^{1}$-complete, while the class of regular continua is $\Pi_{4}^{0}$-complete.
\end{abstract}

1. Introduction. In this note we study some natural classes of continua from the viewpoint of descriptive set theory: motivations, style and spirit are the same of papers such as [Dar00], [CDM02], and [Kru03]. Pol and Pol use similar techniques to study problems in continuum theory in [PP00].

By a continuum we always mean a compact and connected metric space. A subcontinuum of a continuum $X$ is a subset of $X$ which is also a continuum. A continuum is nondegenerate if it contains more than one point. A curve is a one-dimensional continuum.

Let us start with the definitions of some classes of continua: all these can be found in $[\mathrm{Nad} 92]$, which is our main reference for continuum theory.

Definition 1.1. A continuum $X$ is hereditarily locally connected if every subcontinuum of $X$ is locally connected, i.e. a Peano continuum.

A continuum $X$ is hereditarily decomposable if every nondegenerate subcontinuum of $X$ is decomposable, i.e. is the union of two proper subcontinua.

A continuum $X$ is regular if every point of $X$ has a neighborhood basis consisting of sets with finite boundary.

A continuum $X$ is rational if every point of $X$ has a neighborhood basis consisting of sets with countable boundary.

The following classes of continua were defined by Lelek in [Lel71].

Definition 1.2. A continuum $X$ is Suslinian if each collection of pairwise disjoint nondegenerate subcontinua of $X$ is countable.

2000 Mathematics Subject Classification: Primary 03E15, 54F15, 54F50, 54H05.

We thank Riccardo Camerlo and Alessandro Andretta for helpful conversations.

Marcone's research was partially supported by INdAM of Italy. 
A continuum $X$ is finitely Suslinian if for every $\varepsilon>0$ each collection of pairwise disjoint subcontinua of $X$ with diameter $\geq \varepsilon$ is finite.

THEOREM 1.3. The following chain of implications for continua holds:

$$
\begin{aligned}
\text { regular } & \Rightarrow \text { finitely Suslinian } \Rightarrow \text { hereditarily locally connected } \\
& \Rightarrow \text { rational } \Rightarrow \text { Suslinian } \Rightarrow \text { hereditarily decomposable } \Rightarrow \text { curve. }
\end{aligned}
$$

Proof. The fact that every hereditarily locally connected continuum is rational, is well known and originally due to Whyburn ([Why42, Theorem V.3.3]). The last implication follows from Mazurkiewicz's theorem (see e.g. [Nad92, Theorem 13.57]) asserting that every compact metric space of dimension at least 2 contains a nondegenerate indecomposable continuum. All other implications are proved by Lelek in [Lel71].

In particular all classes of continua introduced in Definitions 1.1 and 1.2 are classes of curves.

None of the implications of Theorem 1.3 reverses, although Lelek noticed that every planar hereditarily locally connected continuum is finitely Suslinian.

Our goal is to understand the complexity of the notions we just defined. To this end we use the hierarchies of descriptive set theory. We explain briefly how descriptive set theory deals with (classes of) continua.

If $X$ is a compact metric space, we denote by $\mathrm{K}(X)$ the hyperspace of nonempty compact subsets of $X$, equipped with the Vietoris topology which is generated by the Hausdorff metric, denoted by $d_{\mathrm{H}}$. Then $\mathrm{K}(X)$ is compact metric $([\operatorname{Kec} 95, \S 4 . \mathrm{F}]$ or $[\operatorname{Nad} 92$, Chapter IV] $)$. We denote by $\mathrm{C}(X)$ the subset of $\mathrm{K}(X)$ which consists of all subcontinua of $X ; \mathrm{C}(X)$ is closed in $\mathrm{K}(X)$ and, therefore, it is a compact metric space. Denote by $I$ the closed interval $[0,1]$. Every compact metric space, and in particular every continuum, is homeomorphic to a closed subset of the Hilbert cube $I^{\mathbb{N}}$. Hence $C\left(I^{\mathbb{N}}\right)$ is a compact metric space containing a homeomorphic copy of every continuum. Similarly, $\mathrm{C}\left(I^{2}\right)$ is a compact metric space containing a homeomorphic copy of every planar continuum. Therefore, if $\mathcal{P}$ is a class of continua closed under homeomorphisms (as those introduced in Definitions 1.1 and 1.2), it makes sense to identify $\mathcal{P}$ with the set of all subcontinua of $I^{\mathbb{N}}$ belonging to $\mathcal{P}$, so that $\mathcal{P}$ becomes a subset of $\mathcal{C}\left(I^{\mathbb{N}}\right)$. Therefore $\mathcal{P}$ can be studied with the tools and techniques of descriptive set theory, which studies Polish (i.e. separable and completely metrizable) spaces. Similarly, by considering $\mathcal{P} \cap \mathrm{C}\left(I^{2}\right)$ we study the class of planar continua belonging to $\mathcal{P}$. When $\mathcal{P}$ is a class of continua which has been studied for its own sake in continuum theory, as those introduced in Definitions 1.1 and 1.2 (rather than being built ad hoc to exhibit certain descriptive set-theoretic features), we say that $\mathcal{P}$ is 
a natural class (this is obviously a sociological, rather than a mathematical notion).

We recall the basic definitions of the hierarchies of descriptive set theory (for more details see e.g. [Kec95]). If $X$ is a metric space we denote by $\boldsymbol{\Sigma}_{1}^{0}(X)$ the family of open subsets of $X$. Then $\boldsymbol{\Pi}_{n}^{0}(X)$ is the family of all complements of sets in $\boldsymbol{\Sigma}_{n}^{0}(X)$, while, for $n \geq 1, \boldsymbol{\Sigma}_{n+1}^{0}(X)$ is the class of countable unions of elements of $\mathbf{\Pi}_{n}^{0}(X)$. This hierarchy (called the Borel hierarchy) can be continued in the transfinite, to include all Borel subsets of $X$. At the lowest stages, $\mathbf{\Pi}_{1}^{0}(X)$ is the family of closed subsets of $X$, while $\boldsymbol{\Sigma}_{2}^{0}(X)$ and $\boldsymbol{\Pi}_{2}^{0}(X)$ are respectively the $F_{\sigma}$ and $G_{\delta}$ subsets of $X$. We will also be interested in sets which are in $\boldsymbol{\Pi}_{4}^{0}$ : in the classical notation these are $G_{\delta \sigma \delta}$ sets. We then denote by $\Sigma_{1}^{1}(X)$ the family of analytic subsets of $X$, i.e. of continuous images of a Polish space. $\Pi_{1}^{1}(X)$ is the class of all complements of sets in $\Sigma_{1}^{1}(X)$, also called coanalytic sets. These families are the first level of the projective hierarchy.

By establishing the position of a set in the Borel and projective hierarchies (i.e. the smallest family to which the set belongs), we obtain some information about the complexity of $\mathcal{P}$. This gives lower limits for the complexity of any characterization of the elements of the set. This also has continuum-theoretic consequences: e.g. a class of continua which is not $\boldsymbol{\Sigma}_{1}^{1}$ does not have a model, i.e. a continuum $M$ such that the continua in the class are exactly the continuous images of $M$.

The main tool for establishing lower bounds on the complexity of a set is Wadge reducibility. If $X$ and $Y$ are metric spaces, $A \subseteq X$, and $B \subseteq Y$, we say that $A$ is Wadge reducible to $B$ (and write $A \leq_{\mathrm{W}} B$ ) if $A=f^{-1}(B)$ for some continuous function $f: X \rightarrow Y$. Notice that if e.g. $B$ is $\Sigma_{n}^{0}$ and $A \leq_{\mathrm{W}} B$ then $A$ is also $\boldsymbol{\Sigma}_{n}^{0}$. Thus, proving that $A \leq_{\mathrm{W}} B$ for some $A$ of known complexity yields a lower bound on the complexity of $B$. If $\boldsymbol{\Gamma}$ is a class of sets in Polish spaces (like the classes $\boldsymbol{\Sigma}_{n}^{0}, \boldsymbol{\Pi}_{n}^{0}, \boldsymbol{\Sigma}_{1}^{1}$ and $\boldsymbol{\Pi}_{1}^{1}$ introduced above), $Y$ is a Polish space and $A \subseteq Y$, we say that $A$ is $\boldsymbol{\Gamma}$-hard if $B \leq_{\mathrm{W}} A$ for every $B \in \boldsymbol{\Gamma}(X)$ where $X$ is a zero-dimensional Polish space. We say that $A$ is $\boldsymbol{\Gamma}$-complete if, in addition, $A \in \boldsymbol{\Gamma}(Y)$. It turns out that a set is $\boldsymbol{\Sigma}_{n}^{0}$-complete if and only if it is $\boldsymbol{\Sigma}_{n}^{0}$ but not $\boldsymbol{\Pi}_{n}^{0}$, and similarly interchanging $\boldsymbol{\Sigma}_{n}^{0}$ and $\boldsymbol{\Pi}_{n}^{0}$. If a set is $\boldsymbol{\Pi}_{1}^{1}$-complete then it is not $\boldsymbol{\Sigma}_{1}^{1}$.

Several natural classes of continua have already been classified according to the hierarchies described above: e.g. the class of hereditarily decomposable continua is $\Pi_{1}^{1}$-complete $([\operatorname{Dar} 00])$. In this paper we show that the classes of hereditarily locally connected, finitely Suslinian and Suslinian continua are each $\Pi_{1}^{1}$-complete (the first two results are proved in Section 2, the latter in Section 3), while the class of regular continua is $\boldsymbol{\Pi}_{4}^{0}$-complete (this is proved in Section 4). It is apparent from the proofs that each of our results applies to planar continua, even if we do not state this explicitly. The result about 
regular continua is noteworthy because natural sets which appear for the first time in the Borel hierarchy at the fourth level are quite rare (and there are no natural examples which appear for the first time at later levels, see [Kec95, p. 189]).

We leave as an open problem the classification of the class of rational continua (it is easily seen to be $\boldsymbol{\Sigma}_{2}^{1}$, i.e. the continuous image of a $\boldsymbol{\Pi}_{1}^{1}$ set, and $\boldsymbol{\Pi}_{1}^{1}$-hard). In the remainder of this section we fix our notation and quote some results we will use frequently.

If $X$ is a metric space, we always denote the metric by $d$. Furthermore, we write $B(p ; \varepsilon)$ for the open ball of center $p \in X$ and radius $\varepsilon>0$, while $\operatorname{diam}(A)$ is the diameter of the set $A \subseteq X$. If $A \subseteq X, \bar{A}$ is the closure of $A$ and $\partial A$ its boundary. We use $\mathbb{N}$ to denote the set of nonnegative integers. $2^{\mathbb{N}}$ is the Cantor space consisting of the infinite sequences of 0's and 1's equipped with the product topology obtained from the discrete topology on $\{0,1\}$; it is a compact metric space homeomorphic to Cantor's middle third set.

We will deal with finite sequences of 0 's and 1 's, which form the set $2^{<\mathbb{N}}$. Let $s, t \in 2^{<\mathbb{N}}$. Then

- $|s|$ is the length of $s$,

- $s 0$ (resp. $s 1$ ) is the sequence of length $|s|+1$ obtained by extending $s$ with a final 0 (resp. 1),

- $s \subseteq t$ means that $s$ is an initial segment of $t$,

- if $n<|s|$ then $s(n)$ is the $(n+1)$ th element of $s$,

- if $n \leq|s|$ then $s\lceil n$ is the initial segment of $s$ which has length $n$, and

- $s \wedge t$ denotes the longest sequence which is an initial segment of both $s$ and $t$.

If $\alpha \in 2^{\mathbb{N}}$, then $s \subset \alpha, \alpha(n)$, and $\alpha\left\lceil n\right.$ also make sense. By $\forall^{\infty} n$ we mean for all but finitely many $n$ 's.

To show that a set $A$ is, say, $\Pi_{1}^{1}$-hard one usually picks an already known $\Pi_{1}^{1}$-complete set $B$, and shows that $B \leq_{\mathrm{W}} A$. Here are the sets we will use in our proofs. $\left(I^{\mathbb{N} \times \mathbb{N}}\right.$ is the space of functions from $\mathbb{N} \times \mathbb{N}$ to $I$, with the product topology.)

Lemma 1.4. (1) Let $D=\left\{\alpha \in 2^{\mathbb{N}} \mid \forall^{\infty} n \quad \alpha(n)=0\right\}$. Then $\left\{C \in \mathrm{K}\left(2^{\mathbb{N}}\right) \mid\right.$ $C \subseteq D\}$ is $\boldsymbol{\Pi}_{1}^{1}$-complete.

(2) The set of all countable compact subsets of $2^{\mathbb{N}}$ is $\boldsymbol{\Pi}_{1}^{1}$-complete.

(3) The set $Q=\left\{\alpha \in I^{\mathbb{N} \times \mathbb{N}} \mid \forall k \forall^{\infty} n \quad \alpha(k, n)<1\right\}$ is $\mathbf{\Pi}_{4}^{0}$-complete.

Proof. (1) and (2) are classical results of Hurewicz (see e.g. [Kec95, Theorem 27.5 and $\S 33 . \mathrm{B}])$.

To prove (3), notice that the results in $[\operatorname{Kec} 95, \S 23 . \mathrm{A}]$ imply that the set

$$
P=\left\{\beta \in 2^{\mathbb{N} \times \mathbb{N} \times \mathbb{N}} \mid \forall k \forall^{\infty} n \exists m \quad \beta(k, n, m)=0\right\}
$$


is $\boldsymbol{\Pi}_{4}^{0}$-complete. Therefore it suffices to show $P \leq_{\mathrm{W}} Q$. To this end define a continuous $f: 2^{\mathbb{N} \times \mathbb{N} \times \mathbb{N}} \rightarrow I^{\mathbb{N} \times \mathbb{N}}$ by $f(\beta)(k, n)=\sum_{m=0}^{\infty} \beta(k, n, m) \cdot 2^{-m-1}$. Checking that $\beta \in P$ if and only if $f(\beta) \in Q$ is straightforward.

In establishing the upper bounds for the classes we study, the following simple fact is useful; for a proof (of a slightly more general version) see [AM97, Lemma 1.3].

Lemma 1.5. Let $X$ be Polish and $Y$ compact metric. If $A \subseteq X \times Y$ is $\boldsymbol{\Sigma}_{2}^{0}$ then $\{x \in X \mid \exists y \in Y \quad(x, y) \in A\}$ is also $\boldsymbol{\Sigma}_{2}^{0}$. Similarly, if $B \subseteq X \times Y$ is $\boldsymbol{\Pi}_{2}^{0}$ then $\{x \in X \mid \forall y \in Y \quad(x, y) \in A\}$ is also $\boldsymbol{\Pi}_{2}^{0}$.

The following facts about subsets of $\mathrm{K}(X)$ are also useful (see [Kec95, Exercise 4.29]).

LEmma 1.6. If $X$ is a metric space, each of the sets $\left\{(K, L) \in \mathrm{K}(X)^{2}\right.$ $K \subseteq L\},\{K \in \mathrm{K}(X) \mid \operatorname{diam}(K) \geq \varepsilon\}$, and $\left\{(K, L) \in \mathrm{K}(X)^{2} \mid K \cap L \neq \emptyset\right\}$ is closed.

Recall also the following well known theorem of continuum theory; for a proof see [Nad92, Theorem 5.4].

Theorem 1.7 (Boundary Bumping Theorem). Let $X$ be a continuum and $U$ a nonempty proper open subset of $X$. If $K$ is a connected component of $\bar{U}$, then $K \cap \partial U \neq \emptyset$.

\section{Hereditarily locally connected continua}

Definition 2.1. Let $X$ be a continuum and $K$ be a nondegenerate subcontinuum of $X$. Then $K$ is a continuum of convergence within $X$ if there exists a sequence $\left\{K_{i}\right\}_{i \in \mathbb{N}}$ of subcontinua of $X$ which converges (in the Vietoris topology) to $K$ and is such that $K_{i} \cap K=\emptyset$ for every $i$.

The following theorem provides a well known characterization of hereditarily locally connected continua (see e.g. [Nad92, Theorem 10.4]).

TheOREM 2.2. A continuum $X$ is hereditarily locally connected if and only if no subcontinuum of $X$ is a continuum of convergence within $X$.

The following fact about continua of convergence will be useful.

Lemma 2.3. Let $X$ be a continuum and $K$ be a continuum of convergence within $X$. If $U$ is open in $K$ and $p$ is such that $p \in U$, there exists $H$ which is a continuum of convergence within $X$ such that $p \in H \subseteq U$.

Proof. Let $V$ be open in $X$ such that $U=V \cap K$, and pick $W$ open in $X$ such that $p \in W$ and $\bar{W} \subseteq V$. Let $\eta>0$ be the distance of $p$ from $\partial W$. Let $\left\{K_{i}\right\}_{i \in \mathbb{N}}$ be a sequence of subcontinua of $X$ which converges to $K$ and is such that $K_{i} \cap K=\emptyset$ for every $i$. 
For every $n \in \mathbb{N}$ there exists $i_{n} \in \mathbb{N}$ such that $K_{i_{n}} \cap B\left(p ; 2^{-n}\right) \neq \emptyset$. Let $p_{n}$ belong to this intersection and let $H_{n}$ be the connected component of $\bar{K}_{i_{n}} \cap W$ which contains $p_{n}$. Notice that the Boundary Bumping Theorem implies that $\operatorname{diam}\left(H_{n}\right) \geq \eta-2^{-n}$. By extracting a subsequence we may assume that $\left\{H_{n}\right\}_{n \in \mathbb{N}}$ converges to some $H$, which is obviously a subcontinuum of $X$.

Notice that $\operatorname{diam}(H) \geq \eta$, and hence $H$ is nondegenerate. Since $H_{n} \subseteq$ $K_{i_{n}}$, we have $H \subseteq K$ and $H_{n} \cap H=\emptyset$, so that $H$ is a continuum of convergence within $X$. Since $d\left(p, H_{n}\right) \leq d\left(p, p_{n}\right)<2^{-n}$ we have $p \in H$. Since $H_{n} \subseteq \bar{W}$ we have $H \subseteq \bar{W}$ and hence $H \subseteq V$; hence $H \subseteq V \cap K=U$.

To prove that the class of planar hereditarily locally connected continua is $\boldsymbol{\Pi}_{1}^{1}$-hard we use a modification of the main argument in [Dar00]. Actually Lemma 2.11 gives another proof (more complicated than the original one) of Darji's main result, i.e. the $\boldsymbol{\Pi}_{1}^{1}$-hardness of the class of planar hereditarily decomposable continua. We start with some definitions: some of these are minor variants of the definitions used in [Dar00].

Definition 2.4. A finite list $\mathcal{G}=\left\langle G_{0}, \ldots, G_{n}\right\rangle$ of sets is a chain if $G_{i} \cap G_{j} \neq \emptyset$ if and only if $|i-j| \leq 1$. Any $G_{i}$ is called a link of $\mathcal{G}$ and we write (with slight abuse of notation) $G_{i} \in \mathcal{G}$.

If the $G_{i}$ 's are subsets of a metric space, the mesh of $\mathcal{G}$, denoted by $\operatorname{mesh}(\mathcal{G})$, is $\max \left\{\operatorname{diam}\left(G_{i}\right) \mid i \leq n\right\}$.

Definition 2.5. A finite collection $\mathcal{G}$ of sets is coherent if $\bigcup \mathcal{G}^{\prime} \cap \bigcup \mathcal{G}^{\prime \prime} \neq$ $\emptyset$ whenever $\mathcal{G}=\mathcal{G}^{\prime} \cup \mathcal{G}^{\prime \prime}$ and $\mathcal{G}^{\prime}, \mathcal{G}^{\prime \prime} \neq \emptyset$.

The following lemma is folklore, and besides an easy exercise.

Lemma 2.6. Let $\mathcal{G}$ be a coherent collection of sets and let $a, b \in \cup \mathcal{G}$. Then there exists a chain $\mathcal{H}$ whose links are elements of $\mathcal{G}$ such that $a, b \in$ $\cup \mathcal{H}$.

Definition 2.7. If $\mathcal{H}=\left\langle H_{0}, \ldots, H_{n^{\prime}}\right\rangle$ and $\mathcal{G}=\left\langle G_{0}, \ldots, G_{n}\right\rangle$ are chains of subsets of a topological space we say that:

- $\mathcal{H}$ is a subchain of $\mathcal{G}$, denoted by $\mathcal{H} \subseteq \mathcal{G}$, if all links of $\mathcal{H}$ are links of $\mathcal{G}$ (and hence there exists $i$ such that $\mathcal{H}$ is either $\left\langle G_{i}, \ldots, G_{i+n^{\prime}}\right\rangle$ or $\left.\left\langle G_{i+n^{\prime}}, \ldots, G_{i}\right\rangle\right)$;

- $\mathcal{H}$ refines $\mathcal{G}$, denoted by $\mathcal{H} \ll \mathcal{G}$, if the closure of every link of $\mathcal{H}$ is contained in some link of $\mathcal{G}$ and if for every $G \in \mathcal{G}$ there exists $H \in \mathcal{H}$ such that $H \cap \bigcup(\mathcal{G} \backslash\{G\})=\emptyset$;

- $\mathcal{H}$ goes straight through $\mathcal{G}$, denoted by $\mathcal{H} \ll_{\mathrm{s}} \mathcal{G}$, if $\mathcal{H} \ll \mathcal{G}, H_{0} \subseteq G_{0}$, and for each $i \leq n$ there exist $j_{0}^{\prime} \leq i_{0}^{\prime} \leq i_{1}^{\prime} \leq j_{1}^{\prime} \leq n^{\prime}$ such that 
○ $H_{i^{\prime}} \cap G_{i} \neq \emptyset$ if and only if $j_{0}^{\prime} \leq i^{\prime} \leq j_{1}^{\prime}$;

○ $H_{i^{\prime}} \subseteq G_{i}$ if and only if $i_{0}^{\prime} \leq i^{\prime} \leq i_{1}^{\prime}$;

in this case the chain $\left\langle H_{i_{0}^{\prime}}, \ldots, H_{i_{1}^{\prime}}\right\rangle \subseteq \mathcal{H}$ is the pass of $\mathcal{H}$ through $G_{i}$;

- $\mathcal{H}$ follows z-pattern through $\mathcal{G}$, denoted by $\mathcal{H} \ll_{\mathrm{z}} \mathcal{G}$ if $\mathcal{H} \ll \mathcal{G}$ and there exist $0<i^{\prime}<j^{\prime}<n^{\prime}$ such that $\left\langle H_{0}, \ldots, H_{i^{\prime}}\right\rangle \ll_{\mathrm{s}} \mathcal{G},\left\langle H_{j^{\prime}}, H_{j^{\prime}-1}, \ldots, H_{i^{\prime}}\right\rangle$ $\ll_{\mathrm{s}} \mathcal{G}$, and $\left\langle H_{j^{\prime}}, H_{j^{\prime}+1}, \ldots, H_{n^{\prime}}\right\rangle \ll_{\mathrm{s}} \mathcal{G}$.

Our definition of $\mathcal{H} \ll_{\mathrm{s}} \mathcal{G}$ is more restrictive than the one of [Dar00], to allow for a precise definition of the pass of $\mathcal{H}$ through $G_{i}$ (a notion that Darji did not need in his earlier paper). Notice that if $\mathcal{H} \ll_{\mathrm{z}} \mathcal{G}$ then $\mathcal{H}$ has three (or two, if $i \in\{0, n\}$ ) passes through $G_{i}$.

Lemma 2.8. Suppose $\left\{\mathcal{G}_{n}\right\}_{n \in \mathbb{N}}$ is a sequence of chains of open sets in a metric space such that

- some $\mathcal{G}_{n}$ has at least two links,

- $\lim _{n \rightarrow \infty} \operatorname{mesh}\left(\mathcal{G}_{n}\right)=0$,

- each link of $\mathcal{G}_{n}$ is connected,

- $\mathcal{G}_{n+1} \ll_{\mathrm{s}} \mathcal{G}_{n}$ for all $n$.

Then $\bigcap_{n}\left(\bigcup \mathcal{G}_{n}\right)$ is an arc.

Proof. This lemma (usually for the less restrictive notion of $\ll_{\mathrm{s}}$ ) is well known and its proof is essentially contained in the proof of Theorem 1 of [Moo62] on p. 84.

Lemma 2.9. Suppose $\left\{\mathcal{G}_{n}\right\}_{n \in \mathbb{N}}$ is a sequence of chains of open sets in a metric space such that

- $\lim _{n \rightarrow \infty} \operatorname{mesh}\left(\mathcal{G}_{n}\right)=0$,

- each link of $\mathcal{G}_{n}$ is connected,

- $\mathcal{G}_{n+1} \ll \mathcal{G}_{n}$ for all $n$,

- $\mathcal{G}_{n+1} \ll_{\mathrm{z}} \mathcal{G}_{n}$ for infinitely many n's.

Then $\bigcap_{n}\left(\bigcup \mathcal{G}_{n}\right)$ is a nondegenerate indecomposable continuum.

Proof. This follows (for the less restrictive notion of $\ll_{\mathrm{z}}$, which employs the less restrictive notion of $\ll_{\mathrm{s}}$ ) from the characterization of indecomposable continua given in [IC68].

Definition 2.10. If $\varepsilon>0$ and $\mathcal{G}$ and $\mathcal{H}$ are chains in a metric space we say that $\mathcal{G}$ and $\mathcal{H}$ are $\varepsilon$-entangled, denoted by $\mathcal{G} \bowtie_{\varepsilon} \mathcal{H}$, if the first link and the last link of $\mathcal{G}$ and $\mathcal{H}$ coincide, and whenever $\mathcal{K} \subseteq \mathcal{G} \cup \mathcal{H}$ is a chain with $\operatorname{diam}(\bigcup \mathcal{K}) \geq \varepsilon, \mathcal{K} \cap \mathcal{G} \cap \mathcal{H} \neq \emptyset$.

LEMma 2.11. Any set of hereditarily decomposable continua which contains all planar hereditarily locally connected continua is $\boldsymbol{\Pi}_{1}^{1}$-hard. 
Proof. Recall the notation of Lemma 1.4(1). We will define $F: \mathrm{K}\left(2^{\mathbb{N}}\right) \rightarrow$ $\mathrm{C}\left(I^{2}\right)$ continuous and such that if $C \subseteq D$ then $F(C)$ is hereditarily locally connected, while if $C \nsubseteq D$ then $F(C)$ is not hereditarily decomposable.

To define $F$ we construct sequences $\left\{\mathcal{G}_{s} \mid s \in 2^{<\mathbb{N}}\right\}$ and $\left\{\mathcal{I}_{s, n} \mid s \in 2^{<\mathbb{N}}\right.$, $n>|s|\}$ such that the following conditions are satisfied:

(1) $\mathcal{G}_{s}$ is a chain of open and connected subsets of $I^{2}$;

(2) $\operatorname{mesh}\left(\mathcal{G}_{s}\right)<2^{-|s|-1}$;

(3) $\mathcal{G}_{s 0} \ll_{\mathrm{s}} \mathcal{G}_{s}$ and $\mathcal{G}_{s 1} \ll_{\mathrm{z}} \mathcal{G}_{s}$;

(4) $\mathcal{G}_{s 0} \bowtie_{2-|s|} \mathcal{G}_{s 1}$;

(5) if $t \neq t^{\prime}$ both have length $n$ and $s=t \wedge t^{\prime}$ then $\mathcal{G}_{t} \cap \mathcal{G}_{t^{\prime}}=\mathcal{I}_{s, n}$;

(6) for every $n>|s|$ and $L \in \mathcal{I}_{s, n}$ there exists $\mathcal{L} \subseteq \mathcal{I}_{s, n+1}$ such that $\overline{L^{\prime}} \subseteq L$ for every $L^{\prime} \in \mathcal{L}$, and for every $t \supset s$ with $|t|=n+1$ the first and last link of each pass of $\mathcal{G}_{t}$ through $L$ belong to $\mathcal{L}$.

Figure 1 pictures the first stages of this construction. Sets delimited by dashed lines are the elements of $\mathcal{G}_{\emptyset}$, while the two chains of smaller sets are $\mathcal{G}_{0}$ and $\mathcal{G}_{1}$. The sets filled with gray are the elements of $\mathcal{I}_{\emptyset, 1}$.

Notice that condition (4) implies that $\mathcal{G}_{s}$ has at least two links whenever $|s|>0$, and that by condition (5) we have $\mathcal{I}_{s, n} \subseteq \mathcal{G}_{t}$ for every $t \supset s$ with $|t|=n$.

If $\alpha \in 2^{\mathbb{N}}$ let $M_{\alpha}=\bigcap_{n}\left(\bigcup \mathcal{G}_{\alpha \mid n}\right)$. Lemmas 2.8 and 2.9 and conditions (1)-(3) imply that if $\alpha \in D$ then $M_{\alpha}$ is an arc, while if $\alpha \notin D$ then $M_{\alpha}$ is indecomposable.

Before defining $F$ we establish two claims about our construction.

Claim 2.11.1. Let $s \in 2^{<\mathbb{N}}, n>|s|$ and $\mathcal{H}$ be a chain such that $\operatorname{diam}(\bigcup \mathcal{H})$ $>2^{-|s|}$ and $\mathcal{H} \subseteq \bigcup\left\{\mathcal{G}_{t}|| t \mid=n \& s \subset t\right\}$. Then $\mathcal{H} \cap \mathcal{I}_{s, n} \neq \emptyset$.

Proof. We argue by induction on $n$. The base case is $n=|s|+1$ and follows from conditions (4) and (5). Suppose the claim holds for $n \geq|s|+1$ and let $\mathcal{H} \subseteq \bigcup\left\{\mathcal{G}_{t}|| t \mid=n+1 \& s \subset t\right\}$ be a chain with $\operatorname{diam}(\bigcup \mathcal{H})>2^{-|s|}$. Let

$$
\mathcal{H}^{\prime}=\left\{L^{\prime} \in \bigcup\left\{\mathcal{G}_{t^{\prime}}|| t^{\prime} \mid=n \& s \subset t^{\prime}\right\} \mid \exists L \in \mathcal{H} \bar{L} \subset L^{\prime}\right\} .
$$

Clearly $\operatorname{diam}\left(\bigcup \mathcal{H}^{\prime}\right) \geq \operatorname{diam}(\bigcup \mathcal{H})>2^{-|s|}$ so that there exist $a, b \in \cup \mathcal{H}^{\prime}$ with $d(a, b)>2^{-|s|}$. Since $\mathcal{H}$ is a chain, $\mathcal{H}^{\prime}$ is coherent and by Lemma 2.6 there exists a chain $\mathcal{H}^{\prime \prime} \subseteq \mathcal{H}^{\prime}$ such that $a, b \in \bigcup \mathcal{H}^{\prime \prime}$ and hence $\operatorname{diam}\left(\cup \mathcal{H}^{\prime \prime}\right)>2^{-|s|}$. By induction hypothesis there exists $L^{\prime} \in \mathcal{H}^{\prime \prime} \cap \mathcal{I}_{s, n}$. Since $L^{\prime} \in \mathcal{H}^{\prime}$ there exists $L \in \mathcal{H}$ with $\bar{L} \subset L^{\prime}$. Since $\operatorname{diam}(\bigcup \mathcal{H})>2^{-|s|}>2^{-n-1}$, by condition (2) there are links of $\mathcal{H}$ which are not contained in $L^{\prime}$. By condition (6) there exists a link in $\mathcal{H} \cap \mathcal{I}_{s, n+1}$.

Claim 2.11.2. Let $s \in 2^{<\mathbb{N}}$ and $X \subseteq \bigcup_{\alpha \supset s} M_{\alpha}$ be a continuum with $\operatorname{diam}(X)>2^{-|s|}$. Then $X \cap M_{\alpha} \neq \emptyset$ for every $\alpha \supset s$. 


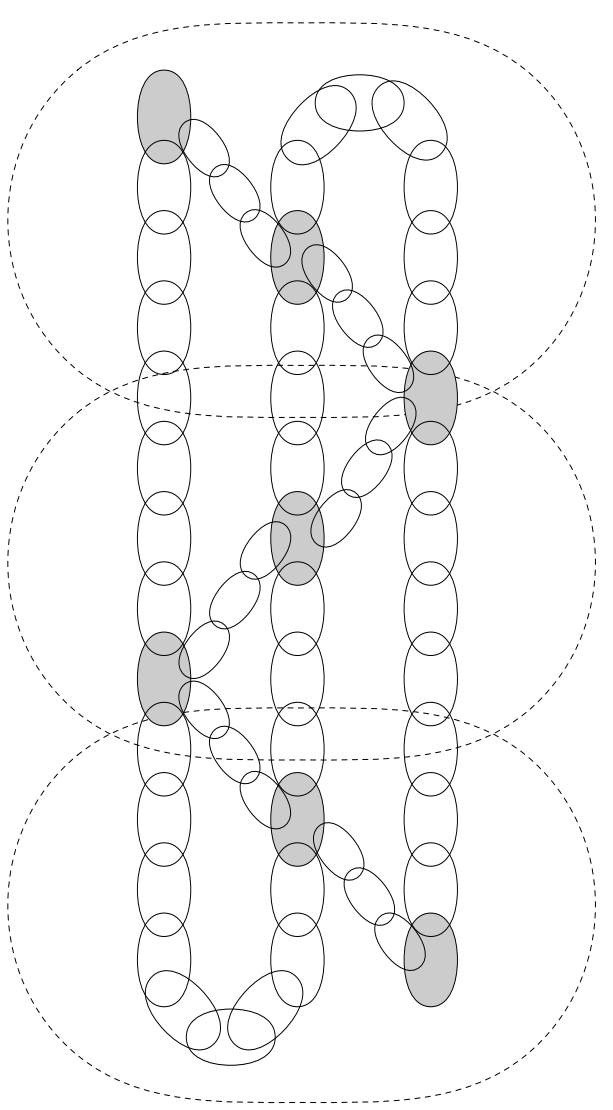

Fig. 1. The construction of Lemma 2.11

Proof. Fix $a, b \in X$ with $d(a, b)>2^{-|s|}$. For every $n>|s|$ let

$$
\mathcal{K}_{n}=\left\{L \in \bigcup\left\{\mathcal{G}_{t}|| t \mid=n \& s \subset t\right\} \mid L \cap X \neq \emptyset\right\} .
$$

Since $X$ is connected, $\mathcal{K}_{n}$ is coherent and Lemma 2.6 implies that there exists a chain $\mathcal{H}_{n} \subseteq \mathcal{K}_{n}$ with $a, b \in \bigcup \mathcal{H}_{n}$, so that $\operatorname{diam}\left(\bigcup \mathcal{H}_{n}\right)>2^{-|s|}$. By Claim 2.11.1 there exists $L_{n} \in \mathcal{H}_{n} \cap \mathcal{I}_{s, n}$. Let $p_{n} \in L_{n} \cap X$. Since $L_{n} \in \mathcal{G}_{\alpha \mid n}$, condition (2) implies that $d\left(p_{n}, M_{\alpha}\right)<2^{-n-1}$. Some subsequence of $\left\{p_{n}\right\}$ converges to some $p$, and clearly $p \in X \cap M_{\alpha}$.

We now define $F: \mathrm{K}\left(2^{\mathbb{N}}\right) \rightarrow \mathrm{C}\left(I^{2}\right)$ by setting $F(C)=\bigcup_{\alpha \in C} M_{\alpha}$. To check that $F$ is continuous it suffices to show that $F(C)=\bigcap_{n} \bigcup_{\alpha \in C}\left(\bigcup \mathcal{G}_{\alpha \mid n}\right)$ and use condition (2). One inclusion is trivial, while for the other let $p \in$ $\bigcap_{n} \bigcup_{\alpha \in C}\left(\bigcup \mathcal{G}_{\alpha \mid n}\right)$. For every $n$ we have $p \in \bigcup \mathcal{G}_{\alpha_{n}\lceil n}$ for some $\alpha_{n} \in C$. There exists a subsequence $\left\{\alpha_{n_{k}}\right\}$ which converges to some $\alpha \in C$. We claim that $p \in M_{\alpha} \subseteq F(C)$, so that we need to show that $p \in \bigcup \mathcal{G}_{\alpha \uparrow n}$ 
for every $n$. Given $n$, pick $k$ such that $n_{k} \geq n$ and $\alpha_{n_{k}}\lceil n=\alpha\lceil n$; then $p \in \bigcup \mathcal{G}_{\alpha_{n_{k}} \mid n_{k}} \subseteq \bigcup \mathcal{G}_{\alpha_{n_{k}} \uparrow n}=\bigcup \mathcal{G}_{\alpha_{n}\lceil n}$.

We now show that $F$ has the properties stated at the beginning of the proof.

If $C \nsubseteq D$ then some indecomposable $M_{\alpha}$ is contained in $F(C)$, and therefore $F(C)$ is not hereditarily decomposable.

Now suppose that $C \subseteq D$; we need to prove that $F(C)$ is hereditarily locally connected. To this end, by Theorem 2.2, it suffices to show that $F(C)$ contains no continuum of convergence. Towards a contradiction suppose $K$ is a continuum of convergence within $F(C)$. Since $K$ is the countable union of the $K \cap M_{\alpha}$ with $\alpha \in C$, for some $\alpha \in C, K \cap M_{\alpha}$ is not nowhere dense in $K$ and there exists $U \subseteq K \cap M_{\alpha}$ open in $K$. By Lemma 2.3 there exists $H \subseteq U$ which is a continuum of convergence within $F(C)$. In particular $H$ is a subarc of $M_{\alpha}$ and there exist $\varepsilon>0$ and $p \in H$ such that $\overline{B(p ; \varepsilon)} \cap M_{\alpha} \subset H$; we may assume $\varepsilon<\frac{1}{3} \operatorname{diam}(H)$. Let $m$ be such that $2^{-m+1}<\varepsilon$ and write $s=\alpha\left\lceil m\right.$. Let $\left\{H_{i}\right\}_{i \in \mathbb{N}}$ be a sequence of subcontinua of $F(C)$ which converges to $H$ and is such that $H_{i} \cap H=\emptyset$. Let $i$ be so large that $d_{\mathrm{H}}\left(H, H_{i}\right)<\varepsilon / 2$, $\operatorname{diam}\left(H_{i}\right)>2 \varepsilon$ and $H_{i} \subseteq \bigcup_{\beta \supset s} M_{\beta}$. Pick $x \in H_{i}$ with $d(x, p)<\varepsilon / 2$ and let $J$ be the connected component of $\overline{B(p ; \varepsilon) \cap H_{i}}$ which contains $x$. Since $H_{i} \nsubseteq \nexists B(p ; \varepsilon)$ (because $\operatorname{diam}\left(H_{i}\right)>2 \varepsilon$ ) and $x \in J$, the Boundary Bumping Theorem implies that $\operatorname{diam}(J)>\varepsilon / 2>2^{-m}$. By Claim 2.11.2, $J \cap M_{\alpha} \neq \emptyset$ and-since $J \cap M_{\alpha} \subseteq \overline{B(p ; \varepsilon)} \cap M_{\alpha} \subset H$-we have $J \cap H \neq \emptyset$ and hence $H_{i} \cap H \neq \emptyset$. This contradicts our hypothesis and completes the proof of the lemma.

THEOREM 2.12. The class of hereditarily locally connected continua is $\boldsymbol{\Pi}_{1}^{1}$-complete.

Proof. The class of hereditarily locally connected continua is $\boldsymbol{\Pi}_{1}^{1}$-hard by Lemma 2.11. Since the class of locally connected continua is Borel (in fact $\Pi_{3}^{0}$-complete, according to a classical result of Kuratowski and Mazurkiewicz [Kur31, Maz31]) it is immediate that the class of hereditarily locally connected continua is $\boldsymbol{\Pi}_{1}^{1}$.

Theorem 2.13. The class of finitely Suslinian continua is $\boldsymbol{\Pi}_{1}^{1}$-complete

Proof. Since in the plane hereditarily locally connected and finitely Suslinian continua coincide ([Lel71]), Lemma 2.11 also establishes $\boldsymbol{\Pi}_{1}^{1}$-hardness of the class of finitely Suslinian continua.

If $X$ is a continuum then $X$ is finitely Suslinian if and only if for all $\varepsilon>0$ we have

$$
\begin{aligned}
\forall\left(Y_{n}\right) \in \mathrm{C}\left(I^{\mathbb{N}}\right)^{\mathbb{N}}\left(\forall n\left(Y_{n} \subseteq X \& \operatorname{diam}\left(Y_{n}\right) \geq \varepsilon\right)\right. & \\
\Rightarrow & \left.\exists n, m\left(n \neq m \& Y_{n} \cap Y_{m} \neq \emptyset\right)\right) .
\end{aligned}
$$


By Lemma 1.6 this equivalence shows that the class of finitely Suslinian continua is $\Pi_{1}^{1}$.

3. Suslinian continua. The following lemma is useful in establishing the upper bound for the class of Suslinian continua, and follows from [CL78, $2.1]$.

Lemma 3.1. Every non-Suslinian continuum has a Cantor set of pairwise disjoint nondegenerate subcontinua.

Theorem 3.2. The class of Suslinian continua is $\boldsymbol{\Pi}_{1}^{1}$-complete.

Proof. By Lemma 3.1, we see that for any continuum $X \in \mathrm{C}\left(I^{\mathbb{N}}\right), X$ is Suslinian if and only if

$$
\begin{aligned}
\forall \mathcal{C} \in \mathrm{K}(\mathrm{C}(X))(\mathcal{C} \text { is uncountable } \& \forall C \in \mathcal{C}(\operatorname{diam}(C)>0) \\
\Rightarrow \exists C, D \in \mathcal{C}(C \cap D \neq \emptyset)) .
\end{aligned}
$$

In view of Lemmas 1.5 and 1.6, and the fact that the uncountable compacta of a compact space form a $\boldsymbol{\Sigma}_{1}^{1}$ set (see e.g. [Kec95, Theorem 27.5]), this formula defines a $\Pi_{1}^{1}$ set.

$\boldsymbol{\Pi}_{1}^{1}$-hardness of the class of Suslinian continua can be proved in several ways: it follows from each of Lemma 2.11, the main proof of [Dar00], and Camerlo's dichotomy for $\sigma$-ideals of continua ([Cam03]). A simpler proof is the following: view $2^{\mathbb{N}}$ as a subset of $I \times\{0\}$, let $p=(0,1)$ and to each $K \in \mathrm{K}\left(2^{\mathbb{N}}\right)$ associate the continuum $C(K)$ which is the union of all straight segments joining any $x \in K$ to $p$ (the cone on $K$ ). It is clear that the map $\mathrm{K}\left(2^{\mathbb{N}}\right) \rightarrow \mathrm{C}\left(I^{2}\right)$ we just defined is continuous and that $C(K)$ is Suslinian if and only if $K$ is countable. By Lemma 1.4(2), the set of Suslinian continua is $\Pi_{1}^{1}$-hard.

4. Regular continua. The following lemma collects some useful characterizations of regular continua.

Lemma 4.1. Let $X$ be a continuum. The following conditions are equivalent:

(1) $X$ is regular;

(2) whenever $p, q \in X$ are distinct, there exists a finite set $F$ such that $p$ and $q$ belong to different connected components of $X \backslash F$ (in this case we say that $F$ separates $p$ and $q$ in $X)$;

(3) for every $\varepsilon>0$ there exists $n$ such that every collection of pairwise disjoint subcontinua of $X$ of diameter $\geq \varepsilon$ has size at most $n$.

Proof. (2) is a well known characterization of regular continua ([Nad92, Theorem 10.19]). The equivalence of (1) and (3) is due to Lelek ([Lel71]). 
Lemma 4.2. Any set of hereditarily locally connected continua which contains all planar regular continua is $\boldsymbol{\Pi}_{4}^{0}$-hard.

Proof. We will use the set $Q$ of Lemma 1.4(3). We are going to define a continuous function $I^{\mathbb{N} \times \mathbb{N}} \rightarrow \mathrm{C}\left(I^{2}\right), \alpha \mapsto L_{\alpha}$, such that

(1) if $\alpha \in Q$ then $L_{\alpha}$ is regular;

(2) if $\alpha \notin Q$ then $L_{\alpha}$ is not hereditarily locally connected.

This suffices to prove the lemma.

Each $L_{\alpha}$ is a subcontinuum of the continuum $L$ described by Nadler in [Nad92, Example 10.38] and drawn in Figure 2: $L$ is not hereditarily locally connected and hence not regular.

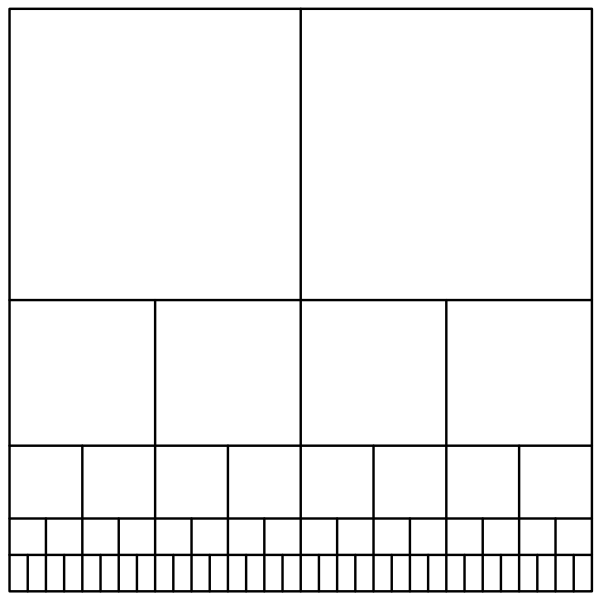

Fig. 2. The continuum $L$

To define $L_{\alpha}$ we need to introduce some notation describing $L$ in some detail (our notation is more detailed than and slightly different from Nadler's). Let $A=I \times\{0\}$ and, for $n \in \mathbb{N}, A_{n}=I \times\left\{2^{-n}\right\}$. For $n \in \mathbb{N}$ and $m \leq 2^{n+1}$ let $B_{n, m}=\left\{\left(m \cdot 2^{-n-1}, y\right) \mid 0 \leq y \leq 2^{-n}\right\}$. Then

$$
L=A \cup \bigcup_{n \in \mathbb{N}} A_{n} \cup \bigcup_{n \in \mathbb{N}} \bigcup_{m \leq 2^{n+1}} B_{n, m} .
$$

For $n \in \mathbb{N}$ and $m<2^{n+1}$, let also

$$
A_{n, m}=\left\{\left(x, 2^{-n}\right) \mid m \cdot 2^{-n-1} \leq x \leq(m+1) \cdot 2^{-n-1}\right\},
$$

so that $A_{n}=\bigcup_{m<2^{n+1}} A_{n, m}$. If $r \in I$, let

$$
A_{n, m}^{r}=\left\{\left(x, 2^{-n}\right) \mid m \cdot 2^{-n-1} \leq x \leq(m+r) \cdot 2^{-n-1}\right\},
$$

so that $A_{n, m}^{r}$ is the left portion of $A_{n, m}$ of length $r$ times the length of the whole segment. For each $n$ and $m<2^{n+1}$, let $k_{n, m}$ be such that $1-2^{-k_{n, m}} \leq$ $m \cdot 2^{-n-1}<1-2^{-k_{n, m}-1}$. 
We can now define $L_{\alpha}$. If $\alpha \in I^{\mathbb{N} \times \mathbb{N}}$ let

$$
L_{\alpha}=A \cup \bigcup_{n \in \mathbb{N}} \bigcup_{m<2^{n+1}-1} A_{n, m}^{\alpha\left(k_{n, m}, n\right)} \cup \bigcup_{n \in \mathbb{N}} \bigcup_{m \leq 2^{n+1}} B_{n, m}
$$

In other words, $L_{\alpha}$ always contains $A$ and the vertical segments contained in $L$, while $\alpha$ dictates how much of the $A_{n, m}$ 's are in $L_{\alpha}$ (notice that we are always leaving out every $\left.A_{n, 2^{n+1}-1}\right)$. A sample $L_{\alpha}$ is drawn in Figure 3 .

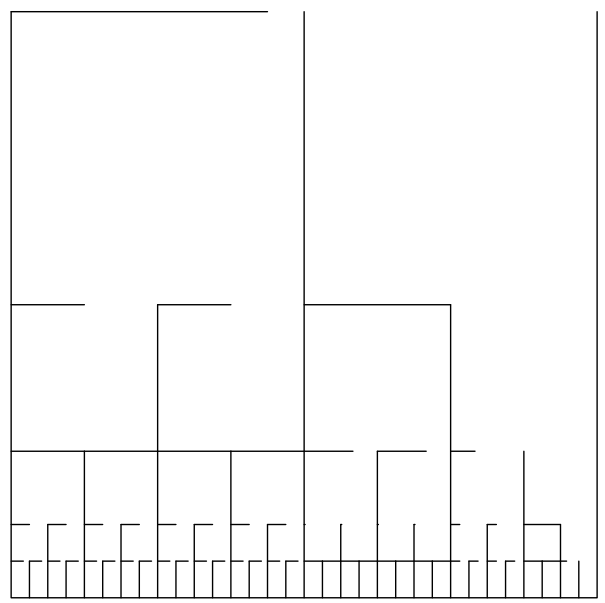

Fig. 3. A sample $L_{\alpha}$ : we are assuming $\alpha(0,0)=7 / 8, \alpha(0,1)=1 / 2, \alpha(1,1)=1$, $\alpha(0,2)=1, \alpha(1,2)=2 / 3, \alpha(2,2)=1 / 3, \alpha(0,3)=1 / 2, \alpha(1,3)=0, \alpha(2,3)=1 / 4$, $\alpha(3,3)=1, \alpha(0,4)=2 / 3, \alpha(1,4)=1, \alpha(2,4)=1 / 2, \alpha(3,4)=1$, and $\alpha(4,4)=1 / 3$.

It is immediate that the function $\alpha \mapsto L_{\alpha}$ from $I^{\mathbb{N} \times \mathbb{N}}$ to $\mathrm{C}(L) \subset \mathrm{C}\left(I^{2}\right)$ is continuous.

In the remainder of the proof we will use implicitly some straightforward observations we summarize here. If $m<2^{n+1}-1$ then the following hold:

- $(m+1) \cdot 2^{-n-1} \leq 1-2^{-k_{n, m}-1}$;

- $k_{n, m} \leq n$;

- $k_{n, m}=n$ if and only if $m=2^{n+1}-2$;

- $m \cdot 2^{-n-1} \leq m^{\prime} \cdot 2^{-n^{\prime}-1}<\left(m^{\prime}+1\right) \cdot 2^{-n^{\prime}-1} \leq(m+1) \cdot 2^{-n-1}$ if and only if $n \leq n^{\prime}$ and $k_{n^{\prime}, m^{\prime}}=k_{n, m}$.

We are now ready to prove (1) and (2).

(1) Suppose $\alpha \in Q$. To prove that $L_{\alpha}$ is regular we will use condition (2) of Lemma 4.1. Let $p$ and $q$ be given. It is immediate that if $p \notin A$ then there exists $F$ containing at most four points which separates $p$ and $q$ in $L$, and a fortiori in $L_{\alpha}$. Thus we may assume that $p, q \in A$, so that $p=(a, 0)$ and $q=(b, 0)$, and furthermore suppose that $a<b$. Let $c=m \cdot 2^{-n-1}$ be such that $a<c<b$, so that $m<2^{n+1}$. Let $k$ be such that $1-2^{-k}<c \leq 1-2^{-k-1}$. 
Since $\alpha \in Q$ there exists $N$ such that $\alpha(k, n)<1$ for every $n>N$. Let

$$
F=\left\{\left(c, 2^{-n}\right) \mid k \leq n \leq N\right\} \cup\{(c, 0)\} .
$$

The connected component of $L_{\alpha} \backslash F$ containing $p$ is $G=L_{\alpha} \cap\{(x, y) \mid x<c\}$ (in fact $\bar{G} \subseteq G \cup F$ ). Thus $p$ and $q$ are in distinct connected components of $L_{\alpha} \backslash F$.

(2) Notice that if $\alpha(k, n)=1$, we have $A_{n, m} \subset L_{\alpha}$ whenever $k_{n, m}=k$ and thus $\left\{\left(x, 2^{-n}\right) \mid 1-2^{-k} \leq x \leq 1-2^{-k-1}\right\} \subset L_{\alpha}$. This shows that if $k$ is such that $\alpha(k, n)=1$ for infinitely many $n$ 's then $\left\{(x, 0) \mid 1-2^{-k} \leq x \leq 1-2^{-k-1}\right\}$ is a continuum of convergence within $L_{\alpha}$. Therefore if $\alpha \notin Q$ then $L_{\alpha}$ contains a continuum of convergence and hence by Theorem 2.2 is not hereditarily locally connected.

TheOREm 4.3. The class of regular continua is $\mathbf{\Pi}_{4}^{0}$-complete.

Proof. The class of regular continua is $\mathbf{\Pi}_{4}^{0}$-hard by Lemma 4.2. By Lemma 4.1 a continuum $X$ is regular if and only if $\forall \varepsilon>0 \exists n \forall\left(C_{0}, \ldots, C_{n}\right) \in \mathrm{C}\left(I^{\mathbb{N}}\right)^{n+1}\left(\forall i \leq n\left(C_{i} \subseteq X \& \operatorname{diam}\left(C_{i}\right) \geq \varepsilon\right)\right.$
$\left.\quad \Rightarrow \exists i, j \leq n\left(i \neq j \& C_{i} \cap C_{j} \neq \emptyset\right)\right)$.

By Lemmas 1.5 and 1.6 this formula defines a $\boldsymbol{\Pi}_{4}^{0}$ set.

Theorem 4.3 implies that we can characterize regular continua by a Borel condition, but this characterization needs to be quite involved.

We like to thank the referee for making valuable changes and improving the exposition of this paper.

\section{References}

[AM97] A. Andretta and A. Marcone, Ordinary differential equations and descriptive set theory: uniqueness and globality of solutions of Cauchy problems in one dimension, Fund. Math. 153 (1997), 157-190.

[Cam03] R. Camerlo, Continua and their $\sigma$-ideals, preprint, 2003.

[CDM02] R. Camerlo, U. B. Darji, and A. Marcone, Classification problems in continuum theory, submitted, 2002.

[CL78] H. Cook and A. Lelek, Weakly confluent mappings and atriodic Suslinian curves, Canad. J. Math. 30 (1978), 32-44.

[Dar00] U. B. Darji, Complexity of hereditarily decomposable continua, Topology Appl. 103 (2000), 243-248.

[IC68] W. T. Ingram and H. Cook, A characterization of indecomposable compact continua, in: Topology Conference (Tempe, AZ, 1967), Arizona State Univ., Tempe, AZ, 1968, 168-169.

[Kec95] A. S. Kechris, Classical Descriptive Set Theory, Grad. Texts in Math. 156, Springer, New York, 1995.

[Kru03] P. Krupski, More non-analytic classes of continua, Topology Appl. 127 (2003), 299-312. 
[Kur31] K. Kuratowski, Évaluation de la classe borélienne ou projective d'un ensemble de points à l'aide des symboles logiques, Fund. Math. 17 (1931), 249-272.

[Lel71] A. Lelek, On the topology of curves. II, ibid. 70 (1971), 131-138.

[Maz31] S. Mazurkiewicz, Sur l'ensemble des continus péaniens, ibid. 17 (1931), 273274 .

[Moo62] R. L. Moore, Foundations of point set theory, revised ed., Amer. Math. Soc. Colloq. Publ. 13, Amer. Math. Soc., Providence, RI, 1962.

[Nad92] S. B. Nadler, Jr., Continuum Theory, Dekker, New York, 1992.

[PP00] E. Pol and R. Pol, On the Krasinkiewicz-Minc theorem concerning countable fans, Houston J. Math. 26 (2000), 869-876.

[Why42] G. T. Whyburn, Analytic Topology, Amer. Math. Soc. Colloq. Publ. 28, Amer. Math. Soc., New York, 1942.

Department of Mathematics

University of Louisville

224 Natural Sciences Building

Louisville, KY 40292, U.S.A.

E-mail: ubdarj01@athena.louisville.edu
Dipartimento di Matematica e Informatica Università di Udine Via delle Scienze 208 33100 Udine, Italy E-mail: marcone@dimi.uniud.it

Received 24 October 2003;

in revised form 21 March 2004 